\title{
Clinical Evaluation of Resin-Based Composites in Posterior Restorations: 12-Month Results
}

\author{
Cigdem Celik \\ Neslihan Arhun ${ }^{b}$ \\ Kivanc Yamanel ${ }^{a}$
}

\begin{abstract}
Objectives: The purpose of this study was to evaluate and compare the 12 month clinical performances of two different posterior composites in Class I and Class II restorations.

Methods: Thirty-one patients ( 10 male, 21 female) were recruited into the study. A total of 82 Class I and Class II cavities were restored with either a nanohybrid composite (Grandio) or a low-shrinkage composite (Quixfil), using their self etch adhesives (Futura Bond and Xeno III) according to manufacturers' instructions. The restorations were clinically evaluated 1 week after placement as baseline, and after 6 and 12 months post-operatively using modified USPHS criteria by two previously calibrated operators. Statistical analysis were performed using Pearson Chi-square and Fisher's Exact Test $(P<.05)$.

Results: All patients attended the 12-month recall. Lack of retention was not observed in any of the restorations. With respect to color match, marginal adaptation, secondary caries and surface texture, no significant differences were found between two restorative materials tested after 12 months $(P>$.05). None of the restorations had marginal discoloration and anatomic form loss on the 12 month follow-up. Restorations did not exhibit post-operative sensitivity at any evaluation period.

Conclusions: Clinical assessment of nanohybrid (Grandio) and low-shrinkage posterior composite (Quixfil) exhibited good clinical results with predominating alpha scores after 12 months. However; further evaluations are necessary for the long-term clinical performance of these materials. (Eur J Dent 2010;4:57-65)
\end{abstract}

Key words: Clinical evaluation; Nanohybrid composites; Posterior composites.

- a Assistant Professor, Baskent University, School of Dentistry, Department of Conservative Dentistry, Ankara, Turkey.

${ }^{b}$ Associate Professor, Baskent University, School of Dentistry, Department of Conservative Dentistry, Ankara, Turkey.

- Corresponding author: Neslihan Arhun Baskent University, Faculty of Dentistry Dept. of Conservative Dentistry

11. Sok No:26 06490 Bahcelievler, Ankara, Turkey. Phone: +90 312 2151336/105 Fax: +90 3122152962 E-mail: neslihanlabaskent.edu.tr

\section{INTRODUCTION}

G. V. Black was the first to describe a systematic method of cavity preparation and the ideal cavity form to restore carious lesions. Classical cavity forms and principles remained appropriate and largely unchallenged for a period of 50 years using amalgam. Amalgam has been the most widely used dental restorative material for the restoration of posterior teeth due to straightforward handling procedures, well tested material properties, 
and clinical success which has been documented for over a century despite esthetic shortcomings. Low material price and rapid application also make it the most economic dental filling material. However, amalgam cavities require precise procedures, usually resulting in uniform depths, particular wall forms, and specific marginal configurations with excessive tooth damage to ensure retention of amalgam.

Thus, patient demand for tooth colored restorations, public concerns related to mercury in dental amalgams, and the desire for a minimally invasive restorations, have made posterior composites an indispensable part of the restorative process instead of amalgam. The increased conservation of healthy dental structure with resin based composite restorations compared to the amalgam ones is the greatest advantage of the former. Many clinicians have used this class of materials quite successfully during the last 5 to 10 years in posterior stress bearing areas. ${ }^{1}$ However, the inherited problems faced using resin based composites were inadequate wear resistance, marginal leakage, secondary caries and lack of appropriate contact. ${ }^{2}$

As manufacturers continue to search for toothcolored resin-based composite materials that will have good physical properties, the introduction of new materials has taken dentistry a step closer to the goal. Recently, a new posterior composite material, Quixfil, was introduced into the dental market. The bimodal filler technology of Quixfil shows particle distribution with two distinct peaks at 0.8 and $10 \mu \mathrm{m}$ and polymerization shrinkage is stated 1.7 vol. $\%$ by the manufacturer. A longitudinal randomized clinical assessment of stress bearing class I and II restorations showed that Quixfil exhibited good clinical results for 3 years. ${ }^{3}$ Nanotechnology may offer unique solutions to improve the performance or handling characteristics of restorative dental materials. Resin composite systems made by the use of nanotechnology can offer high translucency, high polish and polish retention similar to that of microfilled composites while maintaining physical properties and wear equivalent to several hybrid composites. ${ }^{4}$ Grandio was used as one of the first resin composites with incorporated nanofillers beside conventional hybrid type fillers, being called nanohybrid composites. Kramer et al ${ }^{5}$ investigated clinical perfor- mance and margin analysis of Grandio in Class II cavities and stated that they were satisfactory after four years.

As a large number of new improved resin brands are being released to the market, it is important for dentists to be aware of the probable longevity and likely modes of failure in posterior composite restorations. This information is best obtained from randomized controlled trials conducted clinically and in the laboratory for a definitive assessment of dental materials. ${ }^{6}$ Hence, the purpose of this study was to evaluate 12 month clinical performance of a nanohybrid and a lowshrinkage posterior resin composite in Class I and Class II restorations.

\section{MATERIALS AND METHODS}

Thirty-one patients (10 male and 21 female) participated and provided written informed consent to participate in the study. This study was approved by the ethics committee of the Baskent University. The patients' age range was 16 to 60 (Mean: 26). Detailed exclusion and inclusion criteria were as follows:

Inclusion criteria were:

(1) Permanent premolars and molars requiring class I and II for treating primary carious lesions, (2) with at least one neighbouring tooth and in occlusion to antagonistic teeth, (3) good oral hygiene.

Exclusion criteria were:

(1) Patients with fewer than 20 teeth, (2) poor hygiene, (3) heavy bruxism habits, (4) periodontal problems and known allergic reactions against any components of the used materials, (5) pathologic pulpal diagnosis with pain (nonvital), (6) fractured or visibly cracked teeth, (7) defective restorations adjacent or opposing to the tooth, (8) rampant caries, (9) atypical extrinsic staining of teeth or staining of any existing tooth colored restorations.

Each patient had at least one pair of restoration. A total of 82 teeth (41 pairs) were restored with either a nanohybrid resin composite Grandio (Voco GmbH, Germany) and its self-etch adhesive Futurabond NR (Voco, Germany) or a low-shrinkage posterior composite Quixfil (Denstply, Germany) and its self etch adhesive Xenolll (Dentsply, Germany) (Table 1). The distribution of materials and tooth locations were randomized by tossing a coin (Table 2). However, interference in the randomization procedure within patients was per- 
formed in order to equally distribute materials into some important variables such as tooth type and position, restoration class type in such a way that minimized the influence of those factors.

All teeth were treated by one dentist of the research team. The teeth were prepared using conventional instruments and adhesive conservative techniques, appropriate local anesthesia have been achieved preoperatively unless declined by the patient. Cavity preparation was limited to removal of carious tissue. The average facio-lingual width of the cavities was approximately one third of the intercuspal width. Calcium hydroxide (Dycal, Dentsply Caulk, Germanyl was placed where indicated for deep cavities. No beveling was performed. The location of the cervical margins was not recorded. For Class II restorations, the dentists used metal matrix bands (Toefflemire, Tele-

Table 1. Material descriptions, batch numbers and manufacturers of the materials used in the study.

\begin{tabular}{|c|c|c|c|c|}
\hline Material & Material Description & Chemical Composition & Manufacturer & Lot number \\
\hline Futurabond NR & $\begin{array}{l}\text { Dentin Bonding Agent: } \\
\text { Light-curing self-etch bond } \\
\text { reinforced with nanofillers }\end{array}$ & $\begin{array}{l}\text { Liquid A: methacryl } \\
\text { phosphorus acid ester and } \\
\text { carbonic acid modified } \\
\text { methacrylic ester } \\
\text { Liquid B: water, ethanol, } \\
\text { silicon ph= } 1.4\end{array}$ & $\begin{array}{l}\text { Voco GmbH } \\
\text { Germany }\end{array}$ & 610456 \\
\hline Xenolll & $\begin{array}{l}\text { Dentin Bonding Agent Single } \\
\text { step self-etch Fluoride } \\
\text { releasing adhesive }\end{array}$ & $\begin{array}{c}\text { Liquid A: } \\
\text { HEMA, Purified Water, } \\
\text { EthanolUrethane } \\
\text { dimethacrylate resin, BHT, } \\
\text { Highly dispersed silicon } \\
\text { dioxide } \\
\text { Liquid B: Phosphoric acid } \\
\text { modified polymethacrylate } \\
\text { resin, Mono fluoro } \\
\text { phosphazene modified meth- } \\
\text { acrylate resin, UDMA, BHT, } \\
\text { Camphorquinone, Ethyl-4- } \\
\text { dimethylaminobenzoate } \\
\text { ph = 1.4 }\end{array}$ & $\begin{array}{l}\text { Dentsply } \\
\text { Caulk } \\
\text { Germany }\end{array}$ & 0505001099 \\
\hline Grandio & $\begin{array}{c}\text { Resin Composite } \\
\text { Universal Light curing } \\
\text { Nanohybrid resin composite }\end{array}$ & $\begin{array}{c}87 \% \text { w/w (71\% volume) } \\
\text { inorganic nano-hybrid filler, } \\
\text { BisGMA, UDMA, TEGDMA }\end{array}$ & $\begin{array}{l}\text { Voco GmbH } \\
\text { Germany }\end{array}$ & 620492 \\
\hline Quixfil & $\begin{array}{l}\text { Resin Composite } \\
\text { Posterior resin composite }\end{array}$ & $\begin{array}{l}86 \% \text { by weight (66\% volume) } \\
\text { filler load UDMA, TEGDMA, } \\
\text { Di- and trimethacrylate resins } \\
\text { Carboxylic acid modified } \\
\text { dimethacrylate resin, BHT UV } \\
\text { stabilizer } \\
\text { Camphorquinone Ethyl-4- } \\
\text { dimethylaminobenzoate } \\
\text { Silinated strontium aluminum } \\
\text { sodium fluoride phosphate } \\
\text { silcate glass }\end{array}$ & $\begin{array}{l}\text { Dentsply Caulk } \\
\text { Germany }\end{array}$ & 0607001089 \\
\hline
\end{tabular}

HEMA: 2-hydroxyethyl methacrylate; BHT: Butylated hydroxy toluene; TEGDMA: Triethylenglycoldimethacryate; BisGMA: bisphenol-A-diglycidylether dimethacrylate; UDMA: Urethane dimethacrylate 
dyne Waterpik Technologies, USAl and wooden wedges. Saliva isolation was accomplished by cotton rolls and saliva ejectors.

All materials were used according to the respective manufacturers' directions. Placement of resin composites followed the incremental technique (2 $\mathrm{mm}$-thick layers). The resin composite was adapted with a flat faced or elliptical condenser and light cured using a halogen light of 500 $\mathrm{mW} / \mathrm{mm}^{2}$ intensity (Hi-Lux Ultra, Benlioglu, Turkey). The light output of the curing unit was monitored with a light meter (Curing Radiometer Model 100; Demetron Corp, USA)

A post occlusal adjustment was performed with carbon paper and the quality of interproximal contacts and cervical adaptation was checked by means of dental floss and interproximal radiographs. The restorations were finished under water-cooling with fine and super fine diamond points (KG Finishing Kit, Karensen Ltd, Brasil) and rubber polishing kits (Eveflex Polisher, EVE Ernst Vetter $\mathrm{GmbH}$, Germany).

All restorations were clinically evaluated after 1 week (baseline), 6 months and 12 months by 2 investigators using the modified USPHS criteria as first described by Cvar and Ryge ${ }^{7}$ and adapted by Wilson et $\mathrm{al}^{8}$ for retention, color matching, marginal discoloration, marginal adaptation, secondary caries, surface texture, anatomic form and postoperative sensitivity (Table 3 ). The examiners were not involved in the placement of the fillings and were unaware of the materials used in this double-blind study. When disagreement arose during evaluation, the examiners had to reach a consensus. All evaluations were carried out under a dental operating light, using flat surfaced mouth mirrors and dental explorers.

Restorations were scored as follows: alpha represented the ideal clinical situation; Bravo was clinically acceptable; Charlie was clinically unacceptable situations where the restoration had to be replaced. For secondary caries detection bitewing radiographs were also taken at every recall.

Statistical analysis was performed using Pearson Chi-square and Fisher's Exact Test for assessing the difference between the restorative materials $(P<.05)$. Cochran's $Q$ test was also employed for evaluating the difference between examination recalls of the same restorative material.

\section{RESULTS}

At the end of 12 months, all restorations (Grandio or Quixfil) were present and a total of 82 restorations were available for clinical evaluation in 31 patients (Recall rate 100\%). None of the restorations had shown any marginal discoloration and anatomic form loss until the end of the 12 months and no restorations exhibited post-operative sensitivity at any evaluation period.

Summary of clinical findings of Ryge criteria with respect to color match, marginal adaptation, secondary caries and surface texture is shown in Table 4. Mainly, the difference between the restorative materials (Grandio and Quixfil) at the end of 12 months was not statistically significant and demonstrating acceptable clinical performance. At the 6-month recall all the restorations received alpha score with respect to each evaluation criteria.

Nevertheless, there were some statistically different issues regarding evaluation criteria of each material itself between the examination recalls.

The percentages of alpha scores for color match were 95\% ( $n=39$ ) for Grandio restorations and $100 \%$ (n=41) for Quixfil restorations. Two Grandio restorations $(5 \%)$ received bravo score $(P=0.135)$ at 12-month recall.

Four Grandio restorations (10\%) received bravo ratings while 37 restorations (90\%) received alpha ratings for marginal adaptation. This difference

Table 2. Distribution of materials and tooth locations of the restorations.

\begin{tabular}{lcccccc}
\hline & \multicolumn{3}{c}{ Maxillar Arch } & \multicolumn{3}{c}{ Mandibular Arch } \\
Restorative & Premolar & \multicolumn{2}{c}{ Molar } & Premolar & \multicolumn{2}{c}{ Molar } \\
materials & Class II & Class I & Class II & Class II & Class I & Class II \\
\hline Quixfil & 14 & 6 & 3 & 7 & 9 & 2 \\
Grandio & 11 & 8 & 5 & 10 & 3 & 4 \\
TOTAL & 25 & 14 & 8 & 17 & 12 & 6 \\
\hline
\end{tabular}


was found to be statistically significant $(P=0.018)$ between baseline and 12 month recalls. Quixfil restorations' marginal adaptation score was alpha for 40 restorations (98\%) and bravo for 1 restoration $(2 \%)$ at the end of 12 months ( $P=0.368)$.

The evaluation of secondary caries results revealed that 39 restorations (95\%) received alpha scores among Quixfil restorations where 2 restorations $(5 \%)$ received charlie ratings $(P=0.135)$. Therefore, none of the Grandio restorations demonstrated secondary caries after 12 months.

The alpha and bravo scores of Grandio restorations for surface texture were $40(98 \%)$ and $1(2 \%)$, respectively $(P=0.368)$. All the Quixfil restorations received alpha scores in terms of surface texture at 12 months.

\section{DISCUSSION}

Resin composite technology has undergone major developments over the last two decades. However, these developments have been so rapid that long-term clinical data on specific products are rarely available because of regular introduction of "improved" versions. Laboratory tests might provide useful information to the potential performance of a filling material and its' handling, but such tests cannot adequately evaluate the clinical performance of a material or clinical handling characteristics. Besides, in vitro studies cannot answer questions about in vivo longevity of these tooth colored restorations. ${ }^{9}$ Long term results with some of these newly developed materials are lacking and remain controversial as studies report inconsistent clinical results. ${ }^{10,11}$

While USPHS system has served well for clinical evaluation, there are some concerns about the sensitivity of the approach in short term clinical evaluations. The lack of sensitivity of the Ryge system to record small early changes, combined with the continually evolving clinical designs and non standard investigator modifications of the categories, scales, and reporting methods, has created a body of literature that is extremely difficult to

Table 3. Modified USPHS evaluation criteria.

\begin{tabular}{|c|c|}
\hline \multirow{2}{*}{ Retention } & Alfa: No loss of restorative material \\
\hline & Charlie: Any loss of restorative material \\
\hline \multirow{3}{*}{ Color Match } & Alfa: Matches tooth \\
\hline & Bravo: Acceptable mismatch \\
\hline & Charlie: Unacceptable mismatch \\
\hline \multirow{3}{*}{ Marginal Discoloration } & Alfa: No discoloration \\
\hline & Bravo: Discoloration without \\
\hline & Charlie: Discoloration with penetration in pulpal direction \\
\hline \multirow{3}{*}{ Marginal Adaptation } & Alfa: Closely adapted, no visible crevice \\
\hline & Bravo: Visible crevice, explorer will penetrate \\
\hline & Charlie: Crevice in which dentin is exposed \\
\hline \multirow{2}{*}{ Secondary Caries } & Alfa: No caries present \\
\hline & Charlie: Caries present \\
\hline \multirow{3}{*}{ Surface Texture } & Alfa: Enamel-like surface \\
\hline & Bravo: Surface rougher than enamel, clinically acceptable \\
\hline & Charlie: Surface unacceptably rough \\
\hline \multirow{3}{*}{ Anatomic Form } & Alfa: Continuous \\
\hline & Bravo: Slight discontinuity, clinically acceptable \\
\hline & Charlie: Discontinuous, failure \\
\hline \multirow{3}{*}{ Postoperative sensitivity } & Alfa: Not present \\
\hline & Bravo: sensitive but diminishing in intensity \\
\hline & Charlie:constant sensitivity, not diminishing in intensity \\
\hline
\end{tabular}


meaningfully interpret. In many cases, the relative insensitivity of the Ryge methods during short and medium term clinical trials $(<3-5$ years) may be misinterpreted. ${ }^{12}$ However, this system is still being used in the clinical researches to compare these finding with the previous ones that utilize the same system.

The first 6 up to 24 months appear as the critical period for the development of deteriorations. ${ }^{13}$ Mair ${ }^{14}$ evaluated posterior composite restorations over a 10-year period. His data documented a wear rate decreasing after the first years. Inevitably, this study can be criticized that the duration of the study is insufficient to confirm long-term suitability of the tested materials; nevertheless these findings provide an indication of their initial clinical performance.

In the present study, the bonding of the two restorative materials was sufficient to provide adequate retention over 12 months and none of the restoration was lost. The findings of this study were similar to the results of other clinical studies examining the resin restorations for the same evaluation period. ${ }^{15,16}$ However, 2 Quixfil restorations failed after twelve months due to secondary caries and these restorations were replaced.

Post-operative sensitivity seemed to be a problem related to resin composite restorations. Many studies $^{17,18}$ have indicated that up to $30 \%$ of the study populations have reported post-operative sensitivity following the placement of a posterior resin restoration. Self-etch primers make the smear layer part of the hybrid layer, as it dissolves the smear layer, incorporating it into the mixture of collagen fibers and resin monomers. Since the smear layer becomes an integral part of the hybrid layer, low sensitivity response may be the outcome, which was also seen in the present study. ${ }^{19}$

In regard to the clinical performance of selfetch systems, the literature contains contradictory findings, as the bonding effectiveness of these adhesives seems to be material dependent. ${ }^{20,21} \mathrm{~A}$ great variety of self-etch systems are available on the market. They differ in the number of bottles, steps, and acidity of the primer solution, among other factors. A closer analysis of the aforementioned clinical trials ${ }^{20,21}$ reveals that the self etching adhesive with good clinical performance did not belong to the group of "strong" self etching adhesives, but to the group of "mild" self etching adhesives. Futurabond NR's and Xeno III's pH are both 1.4 belonging to the same group.

The loss of marginal adaptation and the presence of secondary caries are predictors of the failure of posterior resin based composites and the reason for the replacement of the restoration. ${ }^{17}$

This study revealed that two Quixfil restorations demonstrated secondary caries although the evaluation period was short. According to Mjör ${ }^{22}$ and Saleh, ${ }^{23}$ development of secondary caries is not only due to the material itself. Clinical environment, caries experience of patients, criteria for replacements, different handling characteristics appeared to affect clinical results. Additionally, Bernardo et $\mathrm{al}^{24}$ reported that the overall risk of failure due to secondary caries was 3.5 times higher in composite restorations than in amalgam restorations.

Grandio restorations have already showed 10\% Bravo scores in terms of marginal adaptation,

Table 4. Summary of the clinical findings of Ryge criteria at the end of 12 months.

\begin{tabular}{lcccccccccccc}
\hline & & \multicolumn{3}{c}{ Color match } & \multicolumn{9}{c}{ Marginal adaptation } & \multicolumn{2}{c}{ Secondary caries } & \multicolumn{3}{c}{ Surface texture } \\
$\begin{array}{l}\text { Grandio+ } \\
\text { Futura Bond }\end{array}$ & n* & A & B & C & A & B & C & A & C & A & B & C \\
\hline Baseline & 41 & $41(100 \%)$ & $0(0 \%)$ & $0(0 \%)$ & $41(100 \%)$ & $0(0 \%)$ & $0(0 \%)$ & $41(100 \%)$ & $0(0 \%)$ & $41(100 \%)$ & $0(0 \%)$ & $0(0 \%)$ \\
6 months & 41 & $41(100 \%)$ & $0(0 \%)$ & $0(0 \%)$ & $41(100 \%)$ & $0(0 \%)$ & $0(0 \%)$ & $41(100 \%)$ & $0(0 \%)$ & $41(100 \%)$ & $0(0 \%)$ & $0(0 \%)$ \\
12 months & 41 & $39(95 \%)$ & $2(5 \%)$ & $0(0 \%)$ & $37(90 \%)$ & $4(10 \%)$ & $0(0 \%)$ & $41(100 \%)$ & $0(0 \%)$ & $40(98 \%)$ & $1(2 \%)$ & $0(0 \%)$ \\
Quixfil + Xeno III & $n^{*}$ & A & B & C & A & B & C & A & C & A & B & C \\
Baseline & 41 & $41(100 \%)$ & $0(0 \%)$ & $0(0 \%)$ & $41(100 \%)$ & $0(0 \%)$ & $0(0 \%)$ & $41(100 \%)$ & $0(0 \%)$ & $41(100 \%)$ & $0(0 \%)$ & $0(0 \%)$ \\
6 months & 41 & $41(100 \%)$ & $0(0 \%)$ & $0(0 \%)$ & $41(100 \%)$ & $0(0 \%)$ & $0(0 \%)$ & $41(100 \%)$ & $0(0 \%)$ & $41(100 \%)$ & $0(0 \%)$ & $0(0 \%)$ \\
12 months & 41 & $41(100 \%)$ & $0(0 \%)$ & $0(0 \%)$ & $40(98 \%)$ & $1(2 \%)$ & $0(0 \%)$ & $39(95 \%)$ & $2(5 \%)$ & $41(100 \%)$ & $0(0 \%)$ & $0(0 \%)$ \\
\hline
\end{tabular}

HEMA: 2-hydroxyethyl methacrylate; BHT: Butylated hydroxy toluene; TEGDMA: Triethylenglycoldimethacryate; BisGMA: bisphenol-A-diglycidylether dimethacrylate; UDMA: Urethane dimethacrylate 
which is statistically significant between baseline and 12 months. Similar to our results, Kramer et $\mathrm{al}^{5}$ found that for marginal adaptation Grandio showed $17 \%$ Bravo scores after one year clinical evaluation period. However, previous researches demonstrated that evaluation of the composites during initial periods of evaluation depicted minor changes compared to the baseline. ${ }^{25,26}$ Marginal adaptation is directly influenced by the type of composite resin used. ${ }^{27}$ Altering the amount and quality of the filler particles can change the esthetics and mechanical properties of restorative composite resins. Furthermore, lowering a material's viscosity by modifying the composition of the monomer system permits a higher filler load and at the same time improves the handling properties. ${ }^{28}$ Grandio has a filler degree of $87 \% \mathrm{w} / \mathrm{w}(71 \%$ volume) by combining spherical nano particles and none of the restorations had shown any marginal discoloration and anatomic form loss until the end of the 12 months and no restorations exhibited post-operative sensitivity at any evaluation period. Quixfil has $86 \%$ by weight (66\% volume) filler load, which is approximately the same.

In a previous study, Manhart et $\mathrm{al}^{29}$ evaluated the clinical performance of Quixfil for 18 months and found significant increase in marginal discoloration with time. While, marginal defects were observed for both materials in our study, none of the restorations showed marginal discoloration. Many of the these marginal defects appeared to result from the fracture of thin flashes of resin composite material extended on non-instrumented enamel surfaces adjacent to the cavity margins. The use of phosphoric acid etching ${ }^{30}$ and aggressive self-etch adhesives ${ }^{32}$ may reduce the occurrence of such defects, especially in highstress-bearing areas, because of the improved enamel etching. ${ }^{30}$ In the present study, mild selfetch adhesive systems were used and marginal adaptation results for 12 months may be related to absence of acid-etching procedure. In consistent to our results, Abdalla and Garcia-Godoy ${ }^{31}$ evaluated the clinical performance of FuturaBond NR in class $\mathrm{V}$ lesions and reported less deteriorations in regards to marginal adaptation and marginal discoloration when adhesive resin was applied following enamel etching.

In the present study, both of the restorative materials demonstrated good color stability and surface texture. At the 1-year recall, the majority of scores were Alfa, Bravo scores were recorded for only two Grandio restorations for color stability and one Grandio restoration for surface texture. However, it has been reported that changes in surface texture and color stability of resin composite restorations could increase after one year. ${ }^{6,33}$

In our study, the greater range of shades was available for Grandio and we expected better color matching ability for this material. Although, Quixfil was available in one universal shade, none of the restorations showed Bravo scores at baseline. Good color match results might be related to chameleon effect of Quixfil, blending into the tooth structure around the restoration.

In the present study, both of these restorative materials were used with their self-etch adhesive systems and demonstrated acceptable clinical performance after 12 months. These successful findings might be related to the relatively short evaluation period, which is consistent with many studies in which there were no significant differences between composite materials in early evaluation periods. ${ }^{15,16}$ It should be noted that the time frame for this study was not of such duration to indicate the long term suitability of the tested materials, but it may provide an indication for detecting material-related initial changes in color and surface topography regarding their future performance.

\section{CONCLUSIONS}

It was concluded that nanohybrid (Grandio) and low-shrinkage posterior composite (Quixfil) demonstrated acceptable clinical performance after 12 months.

\section{REFERENCES}

1. Gerbo L, Leinfelder KF, Mueninghoff L, Russell C. Use of optical standards for determining wear of posterior composite resins. J Esthet Dent 1990;2:148-152.

2. Leinfelder KF. Posterior composite resins: the materials and their clinical performance. $J$ Am Dent Assoc 1995; 126:663-672.

3. Manhart J, Chen HY, Hickel R. Three-year results of a randomized controlled clinical trial of the posterior composite QuiXfil in class I and II cavities. Clin Oral Investig 2008 DOI:10.1007/s00784-008-0233-5) 
4. Mitra SB, Wu D, Holmes BN An application of nanotechnology in advanced dental materials. J Am Dent Assoc 2003;134:1382-1390.

5. Krämer N, Reinelt C, Richter G, Petschelt A, Frankenberger R. Nanohybrid vs. fine hybrid composite in Class II cavities: Clinical results and margin analysis after four years. Dent Mater 2009, doi:10.1016/j.dental.2008.12.003.

6. Fagundes TC, Barata TJ, Bresciani E, Cefaly DF, Jorge MF, Navarro MF. Clinical evaluation of two packable posterior composites: 2-year follow-up. Clin Oral Investig 2006;10:197-203.

7. Cvar JF, Ryge G Criteria for the clinical evaluation of dental restorative materials, US Public Health Service Publication No 790-244 San Francisco: Government Printing Office. 1971

8. Wilson MA, Cowan AJ, Randall RC, Crisp RJ, Wilson NH. A practice-based, randomized, controlled clinical trial of a new resin composite restorative: one-year results. Oper Dent 2002;27:423-429.

9. Efes BG, Dörter C, Gömeç Y. Clinical evaluation of an ormocer, a nanofill composite and a hybrid composite at 2 years. Am J Dent 2006;19:236-240.

10. Loguercio AD, Reis A, Rodrigues Filho LE, Busato AL. Oneyear clinical evaluation of posterior packable resin composite restorations. Oper Dent 2001;26:427-434.

11. Ernst CP, Martin M, Stuff S, Willershausen B. Clinical performance of a packable resin composite for posterior teeth after 3 years. Clin Oral Investig 2001;5:148-155.

12. Hickel R, Roulet JF, Bayne S, Heintze SD, Mjör IA, Peters M, Rousson V, Randall R, Schmalz G, Tyas M, Vanherle G. Recommendations for conducting controlled clinical studies of dental restorative materials. Int Dent J 2007;57:300302

13. Ostlund J, Möller K, Koch G. Amalgam, composite resin and glass ionomer cement in Class II restorations in primary molars--a three year clinical evaluation. Swed Dent J 1992;16:81-86.

14. Mair LH. Ten-year clinical assessment of three posterior resin composites and two amalgams. Quintessence Int 1998;29:483-490.

15. Dresch W, Volpato S, Gomes JC, Ribeiro NR, Reis A, Loguercio AD. Clinical evaluation of a nanofilled composite in posterior teeth: 12-month results. Oper Dent 2006;31:409417.

16. Yip KH, Poon BK, Chu FC, Poon EC, Kong FY, Smales RJ Clinical evaluation of packable and conventional hybrid resin-based composites for posterior restorations in permanent teeth: results at 12 months. J Am Dent Assoc 2003;134:1581-1589.
17. Letzel $H$. Survival rates and reasons for failure of posterior composite restorations in multicentre clinical trial. J Dent 1989;17 Suppl 1:S10-17.

18. Stangel I, Barolet RY. Clinical evaluation of two posterior composite resins: two-year results. J Oral Rehabil 1990;17:257-268

19. Opdam NJ, Roeters FJ, Feilzer AJ, Verdonschot EH. Marginal integrity and postoperative sensitivity in Class 2 resin composite restorations in vivo. J Dent 1998;26:555-562.

20. Türkün S. Clinical evaluation of a self-etching and a onebottle adhesive system at two years. J Dent 2003;31:527534.

21. Van dijken JW. Durability of three simplified adhesive systems in Class $\mathrm{V}$ non-carious cervical dentin lesions. Am J Dent 2004;17:27-32.

22. Mjör IA. Frequency of secondary caries at various anatomical locations. Oper Dent 1985;10:88-92.

23. Saleh N, Peretz B, Rehany A, Zyskin D, Hirschfeld Z, Stark M. One-year clinical evaluation of an anterior composite resin. Quintessence Int 1992;23:559-567.

24. Bernardo M, Luis H, Martin MD, Leroux BG, Rue T, Leitão J, DeRouen TA. Survival and reasons for failure of amalgam versus composite posterior restorations placed in a randomized clinical trial. J Am Dent Assoc 2007;138:775-783.

25. Abdalla Al, Alhadainy HA. 2-year clinical evaluation of Class I posterior composites. Am J Dent 1996;9:150-152.

26. Leinfelder KF. Posterior composite resins: the materials and their clinical performance. J Am Dent Assoc 1995;126:663-664, 667-668, 671-672.

27. Van Meerbeek B, Braem M, Lambrechts P, Vanherle G. Evaluation of two dentin adhesives in cervical lesions. $J$ Prosthet Dent 1993;70:308-314.

28. Närhi TO, Tanner J, Ostela I, Narva K, Nohrström T, Tirri T, Vallittu PK. Anterior Z250 resin composite restorations: one-year evaluation of clinical performance. Clin Oral Investig 2003;7:241-243.

29. Manhart J, Chen HY, Neuerer P, Thiele L, Jaensch B, Hickel R. Clinical performance of the posterior composite QuiXfil after 3,6 , and 18 months in Class 1 and 2 cavities. Quintessence Int 2008;39:757-765.

30. Ferrari M, Mannocci F, Kugel G, García-Godoy F Standardized microscopic evaluation of the bonding mechanism of NRC/Prime \& Bond NT. Am J Dent 1999;12:77-83.

31. Abdalla Al, Garcia-Godoy F. Clinical performance of a selfetch adhesive in Class $\mathrm{V}$ restorations made with and without acid etching. J Dent 2007;35:558-563.

32. Tay FR, Pashley DH, King NM, Carvalho RM, Tsai J, Lai SC, Marquezini L Jr Aggressiveness of self-etch adhesives on unground enamel. Oper Dent 2004;29:309-316. 
33. Loguercio AD, Reis A, Hernandez PA, Macedo RP, Busato AL. 3-Year clinical evaluation of posterior packable composite resin restorations. J Oral Rehabil 2006;33:144-151. 